\title{
Numerical methods for analysis of mechanical systems with uncertain parameters
}

\author{
Milan Vaško ${ }^{1}$, Milan Sága ${ }^{1}$, Alan Vaško ${ }^{2}$ \\ ${ }^{1}$ University of Žilina, Faculty of Mechanical Engineering, Department of Applied Mechanics, Univerzitná 8215/1, 01026 Žilina, Slovakia, \\ phone: +421-41-513 2981,e-mail: milan.vasko@fstroj.uniza.sk, milan.saga@fstroj.uniza.sk \\ 2 University of Žilina, Faculty of Mechanical Engineering, Department of Materials Engineering, Univerzitná 8215/1, 010 26 Žilina, Slova- \\ kia, phone: +421-41-513 2605, e-mail: alan.vasko@fstroj.uniza.sk
}

\begin{abstract}
The paper presents new and improved numerical methods designed for analysis of mechanical systems with uncertain parameters. Uncertain parameters in mechanical systems are described by means of possibility theory. The main representatives of this theory are fuzzy and interval numbers. Algorithms using interval and fuzzy numbers are incorporated into the analysis. The possibilities and efficiency of suggested methods and algorithms are tested by programs created in the environment of the software package MATLAB.
\end{abstract}

Key words - possibility theory, uncertain parameters, interval numbers, fuzzy numbers, numerical methods, MATLAB

\section{Introduction}

The real world features a significant degree of uncertainties. There have been numerous attempts to describe the real phenomena with various means to capture them as accurately as possible. No mathematical model is able to describe all the features of the real system. The problem is to set up a suitable model which would provide us with relevant information about the real situation in mathematical terms as concisely as possible. In technical practice, uncertainties occur in the form of various technical parameters as production and operational parameters, experimentally obtained values and material characteristics, loadings, rounding errors, problems of solution stability, errors of used methods, etc. (TRŠKO L. 2016, ULEWICZ R. 2014). From the viewpoint of mathematical description, uncertainties can be divided into two groups - mathematically inexpressible (verbal), and expressible (applying an appropriate theory).

\section{Analysis of known approaches}

Many numerical methods are used to describe the behaviour of complicated technical systems. The most probable sources of uncertainties in analyses are parameter uncertainties, discretisation errors, rounding error, errors of the method or algorithm, etc.

The Monte Carlo method uses for further analyses properly generated random values. The principle lies in mapping an applied series of analyses for randomly generated uncertain system parameters. It is possible to obtain a map of solution which describes the area of all possible solutions for a given problem.

To solve tasks using the MC method requires a multitude of random numbers. Random numbers are 
generated with the support of the so called generators of random numbers (SÁGA M. 2009, DEKÝŠ V. 2006). The advantage of the MC method is that it is not necessary to know the exact relationships among given and searched values of the task. The most relevant is the use of the method for calculations and optimisation in the field of mechanical engineering, mainly for optimisation of parameters of mechanical systems and constructions (HURTALOVÁ L. 2015, JAGUSIAKKOCIK M. 2014).

Interval analysis is used for calculations with real number intervals. While common calculation is influenced by rounding errors and produces inaccurate results, interval arithmetic has the advantage of precise boundaries for precise solutions. If any parameters are not given precisely but with the interval in which they lie, then the problem can be solved with the algorithm using interval arithmetic (NEUMAIER A. 1990).

If the interval's lower and upper boundaries can be rounded down or up, each of them individually, then, the final exactness of result can be achieved using the interval to express the neighbourhood of exact solution. It is easy to realise it as there are algorithms which use intervals instead of real numbers. The idea of boundary rounding errors using intervals was put into practice in 1950 (ZADEH L.A. 1965). The real interval $\mathbf{x}$ is a non-empty set of real numbers

$$
\mathbf{x}=\langle\underline{x}, \bar{x}\rangle=\{\mathbf{x} \in \mathbf{R}: \underline{x} \leq x \leq \bar{x}\},
$$

where $\underline{x}$ is denoted as infimum and $\bar{x}$ is denoted as supremum. If the following is given

$$
\mathbf{x}=[\underline{x}, \bar{x}] \text { and } \mathbf{y}=[\underline{y}, \bar{y}],
$$

then four basic operations ( $o p)$ are defined:

$$
\mathbf{x} \text { op } \mathbf{y}=\{x \text { op } y: x \in \mathbf{x}, y \in \mathbf{y}\}, \text { op } \in\{+,-, \times, \div\} .
$$

The division by an interval containing zero is not defined for elementary interval operations. This restriction can be removed using the so called extended interval arithmetic. Associative and commutative rules hold for the sum and multiplication, with the exception of special cases because in interval arithmetic the distribution law does not apply (MOORE R.E. 1979).

Fuzzy arithmetic is an efficient aid in solving engineering problems with uncertain parameters. Practical use of standard fuzzy arithmetic seems problematic due to the so called overestimation effect which causes a less or more significant deviation between the arithmetic and calculated problem solution. In general, these deviations can be reduced or completely removed. Numerical tests prove that the implementation of fuzzy arithmetic seems problematic also because some results of the analysis do not include only natural uncertainties (directly induced in the model parameters), but also some other unnatural uncertainties, i.e. generated by the procedure of the solution itself (KAUFMAN A. 1991, HANSS M. 2000).

On the one hand, fuzzy numbers can be implemented as L-R fuzzy numbers. In this form, the number of members in a fuzzy number is characterised by the increasing left-hand side and decreasing right-hand side branch. These are appropriately expressed by parameterised functions belonging to the defined class of basis functions. The fuzzy number type is then referred to as triangular fuzzy number $\mathbf{A}=\left(a_{1}, a_{2}, a_{3}\right)$ or as trapezoidal fuzzy number $\mathbf{B}=\left(b_{1}, b_{2}, b_{3}, b_{4}\right)$. The successful use of fuzzy numbers with any shape of membership functions can be complicated and the evaluation of simple non-linear operations is problematic due to the change in the type of basis functions. The membership functions are given in Fig. 1 (ZHANG H. 2005).

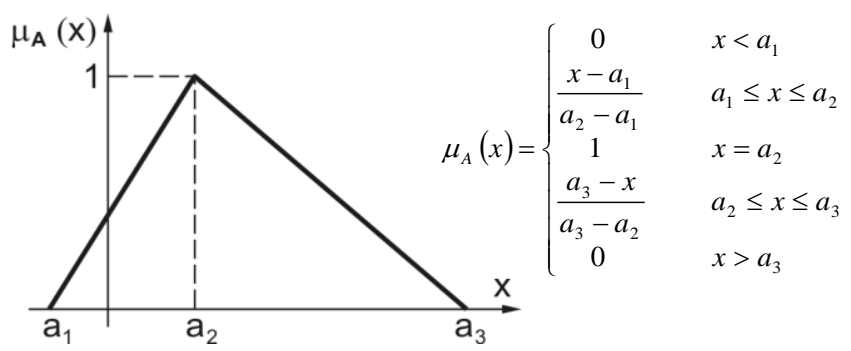

Fig. 1. Fuzzy number $\mathbf{A}$ and membership function. Source: ZHANG H. 2005.

\section{Algorithmisation of computing tech- niques}

The application of interval arithmetic faces the mentioned problem - the overestimation effect. Its elimination is possible only when meeting certain above mentioned assumptions which significantly influence the time efficiency of calculation procedures. The chapter analyses approaches used or proposed by the author and co-authors. 
Monte Carlo method (MC) - the use of the MC is a time consuming but reliable solution procedure. Generating various combinations of deterministic values of uncertain parameters and by the consequent solution accomplished in the deterministic sense, a complete series of results is obtained and properly processed (Fig. 2a). The random matrix of inputs $\mathbf{Q}_{n \times m}$ is generated, $n$ is the number of indefinite inputs and $m$ is the number of generated inputs according to the relation $q_{i} \in\left\langle a_{i} \quad b_{i}\right\rangle$. The matrix $\mathbf{Q}$ will have the form $\mathbf{Q}=\left[\mathbf{q}_{1}, \mathbf{q}_{2}, \ldots, \mathbf{q}_{\mathrm{m}}\right]$. The set of solutions is obtained where the following expression will be for $i$-th solution

$$
\tilde{\mathbf{P}}_{i}=P\left(\mathbf{q}_{i}\right) \text { if } i=1, \ldots, m .
$$

The value determination for the infimum and supremum solutions is

$$
\inf P=\min \tilde{\mathbf{P}} \text { and } \sup P=\max \tilde{\mathbf{P}} \text {. }
$$

Method for evaluation of solution in marginal values (COM1) - it has its physical reasoning for many engineering tasks. The approach is based on the consideration that extreme values at the output are obtained applying the extreme values of parameters for the input (HARGREAVES G.I. 2002). The infimum solution is obtained by the deterministic analysis for all the infima and the supremum is determined by deterministic analysis of the indefinite parameter suprema (Fig. 2b). Two values generated in the solution are at the same time the resultant values, i.e., they represent the resultant infimum and supremum. The vector of possible solutions to $\widetilde{\mathbf{P}}$ is obtained by the deterministic analysis

$$
\widetilde{\mathbf{P}}=P\left[\begin{array}{l}
\mathbf{p}_{\text {inf }} \\
\mathbf{p}_{\text {sup }}
\end{array}\right] .
$$

The searched extreme values of the solution are obtained as in the equation (5). The drawback of this method is that in the process of analysis, significant deviations and inaccuracies in the results occur in comparison with some other methods used for the problem solution.

Method for evaluation of solution for all combinations of marginal values (COM2) - this approach seems to be more suitable. The combinations of the minimum and maximum values from the entire interval solution are also considered (Fig. 2c). The method gives satisfactory results and can be termed as reliable although there may again be a doubt concerning the existence of extreme solution for the internal values. The vector of possible solutions to $\widetilde{\mathbf{P}}$ is also obtained by the deterministic analysis

$$
\widetilde{\mathbf{P}}=P\left(\left[\begin{array}{l}
\mathbf{p}_{\text {inf }} \\
\mathbf{p}_{\text {sup }}
\end{array}\right] \cdot\left[\mathbf{p}_{\text {inf }}^{T}, \mathbf{p}_{\text {sup }}^{T}\right]\right) .
$$

The searched extreme values of the solution are again obtained as in the equation (5). Method is time consuming, which, of course, grows with the number of input values of indefinite parameters in mutual combining their infima and suprema. Yet, it is incomparably less time consuming that the MC.

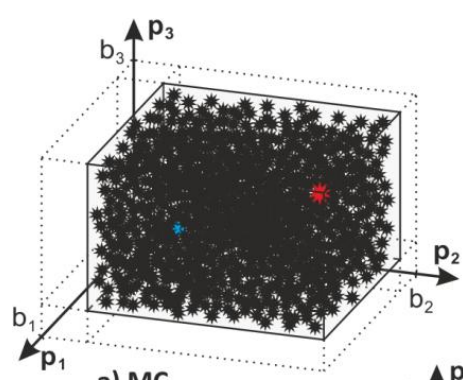

a) MC

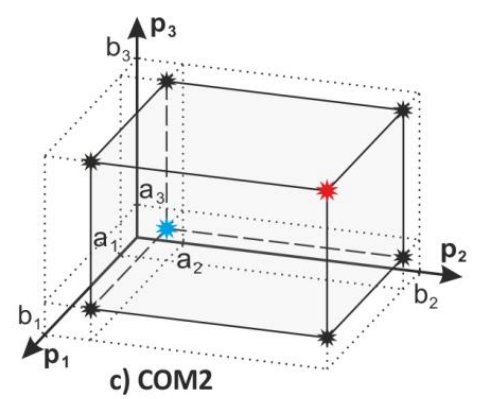

$$
\text { c) } \mathrm{COM} 2
$$

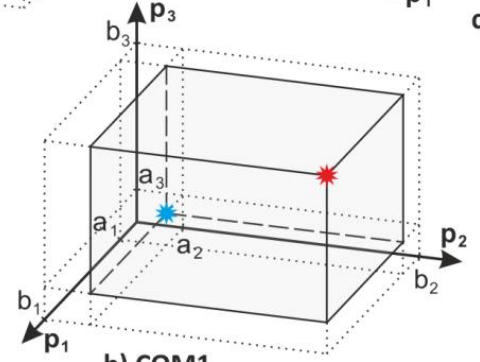

b) COM1

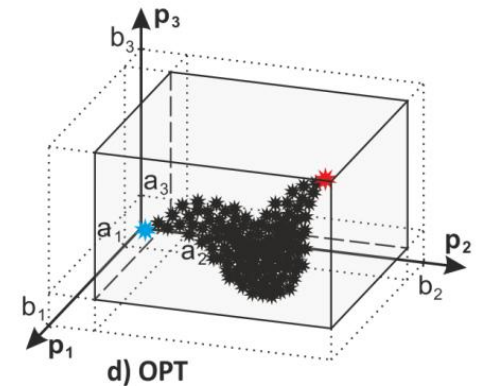

d) OPT

Fig. 2. Set of solutions for the function P using the proposed methods 
Method for seeking inf-sup solution applying the optimising procedure (OPT) - the aim of the method is to reduce a great number of analyses performed in the MC application and, at the same time, to eliminate the problem arising from the possible existence of infimum or supremum from the interior of interval parameters (COM1 and COM2). After the comparison and consideration of individual optimisation methods, it was decided to implement for further analyses the method applying genetic algorithms for optimisation (Fig. 2d). The method is relatively robust, it gives very good results and reliably achieves the global minimum or maximum (VAŠKO M. 2007).

The vector $\mathbf{p}_{\text {inf }}$ that would meet the condition $\mathbf{p}_{\text {inf }} \rightarrow$ min or the vector $\mathbf{p}_{\text {sup }}$ with the condition $\mathbf{p}_{\text {sup }}$ $\rightarrow$ max is being looked for. The advantage of this method is the finding of such combinations of the vector $\mathbf{p}$ members that it is possible to obtain from them, using deterministic analysis, the searched values of infimum and supremum. Then

$$
\inf P=P\left(\mathbf{p}_{\text {inf }}\right) \text { and } \sup P=P\left(\mathbf{p}_{\text {sup }}\right) \text {. }
$$

\section{Conclusion}

The use of fuzzy sets or interval numbers to solve the tasks of engineering mechanics opens new possibilities for quality assessment of mechanical machines. The results either in the fuzzy form or in the form of interval numbers provide a lot of information related to the cumulation of any (even slight) inaccuracies in constructional parameters.

The presented paper points out that even with more numerous indefinite system parameters defined by fuzzy or interval numbers it is possible to achieve the result with a relatively great range of boundary values. This leads to the assumed conclusion that the more indefinite parameters enter the system, the more significant the uncertainty of resultant values is.

\section{Acknowledgements}

This work has been supported by the grant project VEGA No. 1/0234/13.

\section{Literature}

1. DeKÝŠ V., SAPIETOVÁ A., KocúR R. 2006. On the Reliability Estimation of the Conveyer Mechanism Using the Monte Carlo Method. "Machine Dynamics Problems“, Vol. 30, No. 3, p. 58-64.

2. HANSS M. 2000. A Nearly Strict Fuzzy Arithmetic for Solving Problems with Uncertainties. $19^{\text {th }}$ Int. Conf. NAFIPS '2000, Atlanta, USA, p. 439-443.

3. HARgREAVES G.I. 2002. Interval Analysis in MATLAB. "Numerical Analysis Report", 416.

4. Hurtalová L., Tillová E., Chalupová M., BELAN J. 2015. Morphology of intermetallic phases in Al-Si cast alloys and their fracture behaviour. „Prod. Engineer. Archives”, Vol. 6, No. 1, p. 2-5.

5. JAGUSIAK-KOCIK M. 2014. Ensuring continuous improvement processes through standardization in the automotive company. „Production Engineering Archives", Vol. 2, No. 1, p. 12-15.

6. KaUfMAn A., GUPTA M. 1991. Introduction to Fuzzy Arithmetic. Van Nostrand Reinhold, 384 p.

7. MoORE R. E. 1979. Methods and Applications of Interval Analysis. Philadelphia, 200 p.

8. NEUMAIER A. 1990. Interval Methods for Systems of Equations. Cambridge. 272 p.

9. SÁGA M., VAŠKo M. 2009. Solution of Mechanical Systems with Uncertainty Parameters using IFEA. "Communications", Vol. 11/2, p. 19-27.

10. TRŠKO L., BOKU゚VKA O., NovÝ F., LAGO J. 2016. Quality and fatigue characteristics relation. „Production Engineering Archives", Vol. 10, No. 1, p. 9-12.

11. Ulewicz R., Nový F., Mazur M., Szataniak P. 2014. Fatigue properties of the HSLA steel in high and ultra-high cycle region. „Production Engineering Archives", Vol. 4, No. 3, p. 18-21.

12. VAŠKO M. 2007. Application of Genetic Algorithms for Solving of Mechanical Systems with Uncertain Parameters. PhD. thesis. (in Slovak).

13. ZADEH L.A. 1965. Fuzzy sets. "Information and Control", Vol. 8, p. 338-353.

14. Zhang H. 2005. Nondeterministic Linear Static Finite Element Analysis: An Interval Approach. Georgia Institute of Technology, $177 \mathrm{p}$. 